\title{
Fragmentation of thin lenses
}

\author{
R.Ye.Brodskii, T.V.Kulik \\ Institute for Single Crystals, NAS of Ukraine, 60 Lenina av., \\ 61001 Kharkiv, Ukraine
}

\section{Received December 27, 2015}

\begin{abstract}
In the paper it was investigated the case of the quasi-two-dimensional fragmentation fragmentation of the round "lenses" considering dependence of the probability of splitting on energy required for this, i.e. the surface of split area. "lenses" - plates with variable thickness. In the paper "lenses" of several types was investigatet, for each case fragment mass distribution was obtained analytically. In the general case - in parametric form and asymptotics explicitly.
\end{abstract}

Keywords: fragmentation, lenses, plates with variable thickness.

В работе исследован случай квазидвумерной фрагментации - фрагментация круглых "линз" с учётом зависимости вероятности откола от необходимой для этого энергии, т.е. от площади поверхности откола. "Линзы" - пластины переменной толщины. В работе исследованы "линзы" нескольких видов, для каждого случая аналитически получено распределение осколков по массам. В общем случае - в параметрическом виде, а также асимптотики в явном виде.

Фрагментація тонких лінз. Р.Є. Бродсъкий, Т.В. Кулік

у роботі досліджений випадок квазідвовимірної фрагментації - фрагментація круглих "лінз" з урахуванням залежності імовірності відколу від необхідної для цього енергії, тобто від площини поверхні відколу. "Лінзи" - пластини змінної товщини. У роботі досліджені "лінзи" декількох видів, для кожного випадку аналітично отриманий розподіл уламків за масами. В загальному випадку - в параметричному виді, а також асимптотики в явному виді.

\section{Introduction.}

The fragmentation or subdivision of the material - a process commonly used in the technology, as well as one of the most widespread in nature [1]. So wide processes group requires a classification. Fragmentation processes usually classified by the characteristics of the two types. The first characteristic the characteristics of the objects are destroyed, the second - the characteristics of the method of exposure. One of the common characteristics is a dimension of destructing object. There are one-dimensional, two-dimensional and three-dimensional fragmentation [2]. One-dimensional fragmentation occurs, for example, in the case of cutting of the long molecules or destruction of long thin rods. Two-dimensional fragmentation is the fragmentation plates [2] of various shapes. Three-dimensional fragmentation - fragmentation of volumetric bodies [3-10]. By way of exposure fragmentation can by classyfied to "weak" (single-impact) and "strong" (mulfi-impact, long) fragmentation [11]. In the case of single-impact fragmentation the result depends on the initial shape of the sample and the method of the strike, the long 


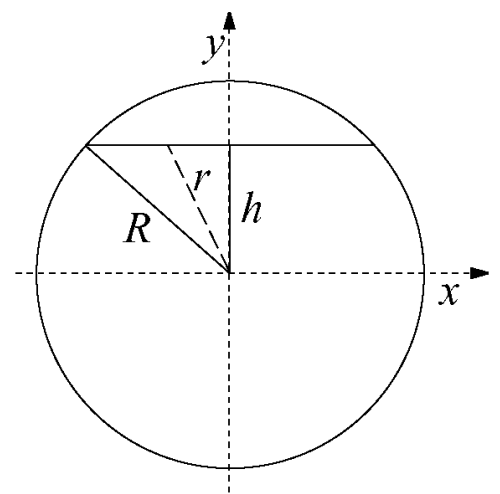

Fig. 1. General designations.

fragmentation often characterized by "forgetting" of initial conditions of the system [12]. Earlier in [13] the fragmentation of circular plates of zero thickness was studied, i.e. two-dimensional fragmentation.

In this paper, the case of the quasi-two-dimentional single-impact fragmentation was studied. For this it was studied the fragmentation of plate of finite thickness, with the thickness of the plates can be either constant or may vary at different points of the plate. Such plates are, generally speaking, lens-like threedimensional bodies. However, if the plate is sufficiently thin, it can be assumed that the plane of split is perpendicular to the plane of the plate, in this case the description of the fragmentation process can be performed in two dimensions. Therefore, this type of fragmentation can be called a quasi-two-dimentional.

The thickness of the plates taked into account as follows. At splitting of a plate of finite thickness break of links in the material on the surface of the split takes place. This decoupling should be consumed energy. It is reasonable to assume that the probability of a split depends on the necessary for this energy, that expended to the formation of a new surface of split and therefore depends on the surface of the split area. This surface area will depend on the position of the split line (line of intersection of the planes of the lens and split). This means that from the probability distribution of the division, which depends on energy, it is possible to obtain the probability distribution of a position of line of split location. And thus, the resulting distribution of fragments by mass.

In the paper the round plate with a centrally symmetric profile thickness was discussed. Those, the plate thickness is a function only of distance from the center, so the plate is a "lens".

Problem formulation. Let's consider the fragmentation of thin circular "lens" with radius $R$. We consider the fragmentation of the plate by the single crack. The crack is direct, i.e. it represents (in the plane of the plate) the chord at distance $h$ from the center. Let's introduce a coordinate system $(x, y)$ with the center in the center of the plate. The plate is central-symmetric, i.e., plate thickness $\eta(x, y)$ is a function only of the distance $r$ from the center of the plate. Hence, the area $s$ of the split surface will dependes only on $h$ and not dependes on the angle of the crack in the specified coordinate system. Hence, for convenience we can orient the coordinate system so that the horizontal axis is parallel to the crack, as shown in Fig.1.

The area of the split surface will be equal to

$$
s(h)=\int_{-\sqrt{R^{2}-h^{2}}}^{\sqrt{R^{2}-h^{2}}} \eta(r(x)) d x
$$

Since the split of plate requires energy proportional to the area of the split surface, the probability of the split by given chord will depend on the need for this energy, i.e. from $s(h)$.

With spalling on chord remoted from the center at a distance $h$, the given fragment mass will be

$$
m(h)=\sigma \int_{h}^{R} s(y) d y
$$


where $\sigma$ is density of the lens material. Thus, knowing the probability distribution of area $s$ of the split surface, we can determine the probability distribution of $h$, and so the distribution of the fragments by mass $m$.

The purpose of this work is to get the mass distribution of the fragments for several "lenses" with desired thickness profiles $\eta(r)$. Namely a convex "lens", concave "lens" and flat "lens" will be considered.

\section{Fragmentation of the convex lens.}

As an example of a convex "lens" let's take the parabolic "lens" - a plate with a thickness profile

$$
\eta(r)=k\left(R^{2}-r^{2}\right)
$$

Such lens shown at Fig.2.

The area of a split surface along the chord at the distance $h$ from the center of this plate will be equal

$$
s(h)=\frac{4}{3} k\left(R^{2}-r^{2}\right)^{3 / 2}
$$

The mass of cutting fragment at this case is

$$
m(h)=\frac{k \sigma R^{4}}{3}\left[\frac{3}{2}\left(\frac{\pi}{2}-\arcsin \frac{h}{R}\right)-\sin \left(2 \arcsin \frac{h}{R}\right)-\frac{1}{8} \sin \left(4 \arcsin \frac{h}{R}\right)\right]
$$

It is important to note that both area $s(h)$ and the fragment mass $m(h)$ decreases monotonically with $h$ (this is clear from simple geometric considerations). From this it follows that if $P_{s}(s)$ - probability distribution of splitting with the formation of the new surface with area $s$, i.e. $\operatorname{Pr}\left\{s \in\left[0, s_{\max }\right]\right\}$, and $P_{m}(m)$ - probability distribution of forming of fragment with mass $m$, i.e. $\operatorname{Pr}\left\{m \in\left[0, m_{\max }\right]\right\}$, then

$$
P_{m}(m)=P_{s}(s(m))
$$

Thus, if we know the distribution $P_{s}(s)$, then in the case of a parabolic lens distribution $P_{m}(m)$ can be obtained from $P_{s}(s)$ and parametric coupling $s(m)$ in the form

$$
\left\{\begin{array}{l}
s(h)=\frac{4}{3} k\left(R^{2}-h^{2}\right)^{3 / 2} \\
m(h)=\frac{k \sigma R^{4}}{3}\left[\frac{3}{2}\left(\frac{\pi}{2}-\arcsin \frac{h}{R}\right)-\sin \left(2 \arcsin \frac{h}{R}\right)-\frac{1}{8} \sin \left(4 \arcsin \frac{h}{R}\right)\right]
\end{array}\right.
$$

And get the distribution density by differentiation of the distribution function as the parametric function.

Let's take

$$
P_{s}(s)=A s^{\alpha}, \quad 0<\alpha<1,
$$

here $A$ - normalization factor. Normalization condition $-P_{s}\left(s_{\max }\right)=1$. The value of $\alpha$ within $(0,1)$ gives the increasing distribution function and as it should be, $P_{s}(0)=0$, and a decreasing function of the density $p_{s}(s)=\frac{d P_{s}(s)}{d s}$. This corresponds to a decrease of the probability of spalling with increasing of energy need for this, that is justified from a physical point of view.

Let's get asymptotics of density $p_{m}(m)=\frac{d P_{m}(m)}{d m}$ for given $P_{s}(s)$ in regions of big and small values of the mass. Big fragment mass, i.e. mass, close to mass of half of the plate $m_{\max }=\frac{k \sigma R^{4}}{3}\left[\frac{3}{2} \cdot \frac{\pi}{2}\right]$ corresponds to small $h, \frac{h}{R}<<1$. In this region

$$
\begin{gathered}
m(h) \approx m_{\max }-\frac{k \sigma R^{4}}{3}\left[\frac{3}{2} \frac{h}{R}+2 \frac{h}{R}+\frac{1}{2} \frac{h}{R}\right]=m_{\max }-4 \frac{k \sigma R^{4}}{3} \frac{h}{R} \\
s(h)=\frac{4}{3} k R^{3}\left(1-\left(\frac{h}{R}\right)^{2}\right)^{3 / 2} \approx \frac{4}{3} k R^{3}\left(1-\frac{3}{2}\left(\frac{h}{R}\right)^{2}\right)
\end{gathered}
$$




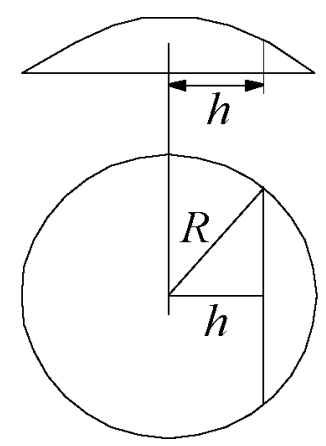

Fig. 2. The parabolic "lens", side and top view. The fault at a distance $h$ from the center of the lens is shown.

And thus

$$
P_{m}(m)=A\left(\frac{4}{3} k R^{3}\left(1-\frac{3}{2}\left(\frac{3}{4} \frac{m_{\max }-m}{k \sigma R^{4}}\right)^{2}\right)\right)^{\alpha}
$$

Let $\alpha=1 / 2$. In this case norming factor

$$
A=s_{\max }^{-1 / 2}=\left(\frac{4}{3} k R^{3}\right)^{-\frac{1}{2}}
$$

and

$$
P_{m}(m)=\left(1-\frac{3}{2}\left(\frac{3}{4} \frac{m_{\max }-m}{k \sigma R^{4}}\right)^{2}\right)^{1 / 2}
$$

So density

$$
p_{m}(m) \approx \frac{3}{2}\left(\frac{3}{4} \frac{1}{k \sigma R^{4}}\right)^{2} \cdot\left(m_{\max }-m\right)
$$

Thus, at big masses region density distribution of the fragments by mass is linear.

Let's get asymptotic for small masses $m<<m_{\max }$. In this region $h \approx R$. Let's inroduse small $\varepsilon$ such that $h=R(1-\varepsilon), \varepsilon<<1$.

Note, however, that in expression for mass

$$
m(h)=\frac{k \sigma R^{4}}{3}\left[\frac{3}{2}\left(\frac{\pi}{2}-\arcsin (1-\varepsilon)\right)-\sin (2 \arcsin (1-\varepsilon))-\frac{1}{8} \sin (4 \arcsin (1-\varepsilon))\right]
$$

which should be expand with a small $\varepsilon$, includes $\arcsin (1-\varepsilon)$, and the derivative of $\arcsin (1-\varepsilon)$ tends to infinity when $\varepsilon \rightarrow 0$. Use as a small parameter $\delta=\sqrt{\varepsilon}$.

Let's expand $s(h)$ and $m(h)$ with small $\delta$.

$$
s(h)=\frac{4}{3} k\left(R^{2}-R^{2}\left(1-\delta^{2}\right)^{2}\right)^{3 / 2} \approx \frac{4}{3} k R^{3}(\sqrt{2} \delta)^{3}
$$

In the expression for the mass all the terms of the expansion up to terms of order $\delta^{4}$ are zero. The first non-zero term in the expansion and, consequently, the asymptotic behavior of the masses, has the form

$$
m(h) \approx \frac{k \sigma R^{4}}{8} \sqrt{2} \times \frac{1039}{120} \delta^{5}
$$

Thus, fragments distribution $P_{m}(m)=P_{s}(s(m))$ with taken above $P_{s}(s)=A s^{-\frac{1}{2}}$ will have the form

$$
P_{m}(m)=(\sqrt{2} \delta)^{3 / 2}, \delta=\delta(m)
$$




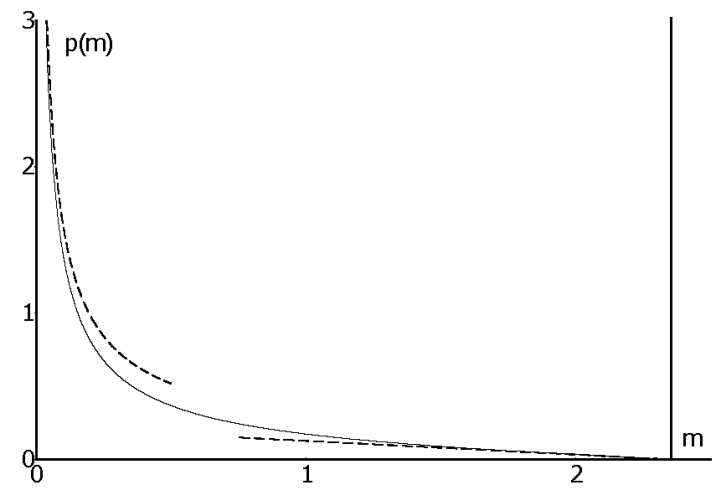

Fig. 3. The distribution density of the fragments by mass and its asymptotic behavior in the case of fragmentation of parabolic convex "lens". Here $\sigma=1, k=3, R=1$.

$$
P_{m}(m)=2^{3 / 4}\left(\frac{k \sigma R^{4}}{8} \frac{1039}{120} \sqrt{2}\right)^{-3 / 10} m^{3 / 10}
$$

From where the distribution density is equal to

$$
p_{m}(m)=2^{3 / 4}\left(\frac{k \sigma R^{4}}{8} \frac{1039}{120} \sqrt{2}\right)^{-3 / 10} \cdot \frac{3}{10} m^{-7 / 10}
$$

Thus, we obtained the asymptotic behavior of the distribution density of the fragments by mass in the case of fragmentation of parabolic convex "lens" explicitly. On Fig. 3 it shows these asymptotics and density graph obtained by differentiating the distribution function $P_{m}(m)$ given parametrically.

Large-scale asymptotic is linear, small-scale is power with exponent $-7 / 10$.

\section{Fragmentation of a flat circular plate.}

Let us now consider a circular flat plate constant zero thickness $\eta$. We will find the distribution and distribution density of the mass of the fragments in the fragmentation of such a plate in the case where the probability of fracture depends on the area of formed surface.

As in the previous case, we assume that the surface of split perpendicular to the plane of the plate, i.e. we are dealing with quasi-two-dimentional fragmentation. Also, as above, we assume that the surface of the split is plane, i.e. in projecttion onto the plane of the plate, we are dealing with cutting a round by chord.

In the case of flat plate area $s$ of split surface and mass $m$ of splinter decrease monotonically as in the previous case, with increasing distance $h$ from the crack-chord to the center of the plate. Thus, the system

$$
\left\{\begin{array}{l}
s=s(h) \\
m=m(h)
\end{array}\right.
$$

sets in parametric form the function $s(m)$. So, with the expression for the probability distribution $P_{s}(s)$ of spalling in depending on the formed surface area, we can get the fragments mass distribution in the form

$$
P_{m}(m)=P_{s}(s(m))
$$

And then get the distribution density, differentiating this expression on $m$.

In the case of a plate with thickness $\eta$ 


$$
\begin{aligned}
& s(h)=2 \eta \sqrt{R^{2}-h^{2}} \\
& m(h)=\sigma \eta R^{2}\left(\frac{\pi}{2}-\arcsin \frac{h}{R}-\frac{h}{R} \sqrt{1-\frac{h^{2}}{R^{2}}}\right)
\end{aligned}
$$

Let's take, as above,

$$
P_{s}(s)=A s^{\alpha}, \quad 0<\alpha<1
$$

$A$ - norming factor, equal to $A=s_{\max }^{-\alpha}$, where $s_{\max }-$ maximal possible section area, $s_{\max }$ corresponds to cutting of plate on diameter and equal to $s_{\max }=s(0)=2 \eta R$.

Expression for $P_{s}(s)=A s^{\alpha}$ and defined above in parametric case function $s(m)$ combine parametrically represented distribution $P_{m}(m)$ of fragments by masses.

Let's obtain asymptotics of distribution density in explicit form for small and big values of mass $m$.

Small $m$ forms at $h \approx R$. Let's write $h=R(1-\delta), \delta<<1$ Then

$$
\begin{gathered}
m(h) \approx \frac{\sigma R^{2} \eta}{\sqrt{2}} \cdot 2 \frac{2}{3} \cdot \delta^{3 / 2} \\
s(h) \approx 2 \eta R \sqrt{2} \sqrt{\delta}
\end{gathered}
$$

To convenience of comparison with other cases we take as above, $\alpha=1 / 2$. In this case

$$
P_{m}(m)=A s^{1 / 2}=(2 \eta R)^{-1 / 2}(2 \eta R \sqrt{2} \sqrt{\delta})^{1 / 2}=2^{1 / 4} \delta^{1 / 4}
$$

Substituting $\delta=\delta(m)$, obtaine

$$
P_{m}(m)=2^{1 / 4}\left(\frac{\sigma R^{2} \eta}{\sqrt{2}} \cdot 2 \frac{2}{3}\right)^{-1 / 6} m^{1 / 6}
$$

differentiating, obtain density

$$
p_{m}(m)=2^{1 / 4}\left(\frac{\sigma R^{2} \eta}{\sqrt{2}} \cdot 2 \frac{2}{3}\right)^{-1 / 6} \frac{1}{6} \cdot m^{-5 / 6}
$$

We now will find the asymptotic behavior for large masses. The large mass of fragments corresponds to a small $h=R \delta \ll R$. In this case

$$
\begin{aligned}
& s(h) \approx 2 \eta R\left(1-\frac{1}{2} \delta^{2}\right) \\
& m(h) \approx \sigma \eta R^{2}\left(\frac{\pi}{2}-2 \delta\right)
\end{aligned}
$$

That is,

$$
P_{m}(m)=P_{s}(s(\delta(m)))=A s^{-1 / 2} \approx 1-\frac{1}{4} \delta^{2}(m)
$$

And, substituting $\delta=\delta(m)$, obtain

$$
P_{m}(m)=\left(1-\frac{1}{4}\left(\frac{\pi}{2}-\frac{m}{\sigma \eta R^{2}}\right)^{2}\right)^{1 / 4}
$$

From where, density

$$
p_{m}(m) \approx \frac{1}{8 \sigma \eta R^{2}}\left(\frac{\pi}{2}-\frac{m}{\sigma \eta R^{2}}\right)
$$

Or denoting $m_{\max }$ maximal possible mass of the fragment $m_{\max }=m(0)=\frac{\pi}{2} \sigma \eta R^{2}$, 




Fig. 4. Distribution density and asymptotics for case of flat plate. Here $\sigma=1, \eta=1, R=1$.

$$
p_{m}(m) \approx \frac{1}{8\left(\sigma \eta R^{2}\right)^{2}}\left(m_{\max }-m\right)
$$

The graph of density obtained by differentiating of parametrically given $P_{m}(m)$, and the asymptotics is given in Fig. 4.

It is evident that found small-scale power and large-scale linear asymptotics fit to the exact density with the great precision in respective areas.

Here and above we choose the distribution of split area in form $P_{s}(s)=A s^{\alpha}$. In [13] the fragmentation of round thin plates was considered, where the probability of spalling on the fracture with a particular position is determined from other (geometric) considerations than in this work. One of the considered cases in this article was a case where a crack-chords were distributed evenly over distance $h$ from the center of the plate, $p_{h}(h)=\frac{1}{R}=$ const.

Let us find the asymptotics of distribution density of the fragments by masses under these conditions, using the approach applied above for $P_{s}(s)=A s^{\alpha}$.

In this case, the distribution function of the chord on $h$ has the form $P_{h}(h)=\frac{h}{R}$, from where, since the area $s$ decreases monotonically with $h$ and $s(h)=2 \eta \sqrt{R^{2}-h^{2}}$, the distribution of the fragments on the split area have the form

$$
P_{s}(s)=1-\sqrt{1-\left(\frac{s}{2 \eta R}\right)^{2}}
$$

Let's obtain the asymptotic behavior of the distribution density of $p_{m}(m)$ for this case.

As it shown above, in small-scale region $h=R(1-\delta), \delta<<1$ we have $s(h) \approx 2 \eta R \sqrt{2} \sqrt{\delta}$. Substituting this expression in $P_{s}(s)$ we obtain

$$
P_{s}(s)=1-\sqrt{1-2 \delta} \approx \delta
$$

Since $P_{m}(m)=P_{s}(s(\delta(m)))$ and in pointed region $m(h) \approx \frac{\sigma R^{2} \eta}{\sqrt{2}} \cdot 2 \frac{2}{3} \cdot \delta^{3 / 2}$, we obtain

$$
P_{m}(m)=\left(\frac{3}{4 \sqrt{2} \sigma R^{2} \eta} m\right)^{\frac{2}{3}}
$$

From where density

$$
p_{m}(m)=\frac{2}{3}\left(\frac{3}{4 \sqrt{2} \sigma R^{2} \eta}\right)^{\frac{2}{3}} m^{-\frac{1}{3}}
$$

or

$$
p_{m}(m)=\left(\frac{1}{2 \sqrt{3} \sigma R^{2} \eta}\right)^{\frac{2}{3}} m^{-\frac{1}{3}}
$$


that coincide with result, obtained in [13].

In large-scale region $h=R \delta \ll R$. In this case $s(h) \approx 2 \eta R\left(1-\frac{1}{2} \delta^{2}\right)$ and

$$
P_{s}(s)=1-\delta
$$

And since $m(h) \approx \sigma \eta R^{2}\left(\frac{\pi}{2}-2 \delta\right)$, then

$$
P_{m}(m)=1-\frac{1}{2}\left(\frac{\pi}{2}-\frac{m}{\sigma \eta R^{2}}\right)
$$

and density

$$
p_{m}(m)=\frac{1}{2 \sigma \eta R^{2}},
$$

that also coincide with result of work [13].

\section{Fragmentation of concave lens.}

We have examined cases of fragmentation of convex ant flat plate. We now consider the fragmentation of the concave parabolic "lens". Let the dependence of the thickness of the "lens" on distance from the center of the plate has the form

$$
\eta(r)=k r^{2}
$$

In this case the function $s(h)$ and $m(h)$ are obtained by subtracting the corresponding expressions for the convex "lens" from the expressions for a flat plate with a thickness of a flat "lens" $\eta=k R^{2}$ (fig.5).

Namely, the split area

$$
s(h)=2 \eta \sqrt{R^{2}-h^{2}}-\frac{4}{3} k\left(R^{2}-h^{2}\right)^{3 / 2}, \quad \eta=k R^{2},
$$

i.e.

$$
s(h)=\frac{2}{3} k \sqrt{R^{2}-h^{2}}\left(R^{2}+2 h^{2}\right)
$$

and fragment mass

$$
\begin{aligned}
& m(h)=\sigma \eta R^{2}\left(\frac{\pi}{2}-\arcsin \frac{h}{R}-\frac{h}{R} \sqrt{1-\frac{h^{2}}{R^{2}}}\right)- \\
& -\frac{k \sigma R^{4}}{3}\left[\frac{3}{2}\left(\frac{\pi}{2}-\arcsin \frac{h}{R}\right)-\sin \left(2 \arcsin \frac{h}{R}\right)-\frac{1}{8} \sin \left(4 \arcsin \frac{h}{R}\right)\right], \eta=k R^{2},
\end{aligned}
$$

i.t.

$$
m(h)=\frac{1}{2} k \sigma R^{4}\left(\frac{\pi}{2}-\arcsin \frac{h}{R}-\frac{2}{3} \frac{h}{R} \sqrt{1-\frac{h^{2}}{R^{2}}}+\frac{1}{12} \sin \left(4 \arcsin \frac{h}{R}\right)\right)
$$

These expressions determine function $s(m)$ in parametric form. The graph of this function given on fig.6.

The key difference from previous cases is that for such a lens the dependence of $s(m)$ not monotonic, and thus the dependence of $m(s)$ ambiguous. That is, the same value of split surface area (and hence the need for chipping energy) may correspond to multiple values of mass fragments.

Thus, equality $P_{m}(m)=P_{s}(s(m))$ in the case of concave "lens" is not realized.

Suppose, as before, that we know the distribution function $P_{s}(s)$. In the case where the same $s$ may correspond to different values of $m$, we need to determine the pribability of these different $m$, if it is known that the area of split is $s$. 


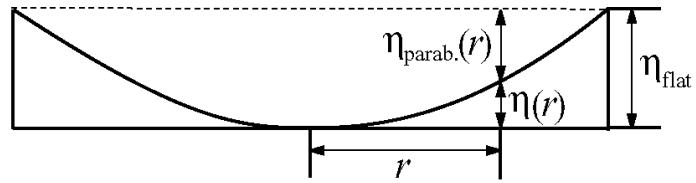

Fig. 5. The concave parabolic "lens". This "lens" can be considered as the result of subtracting the convex parabolic "lens" from the volume of the plate.

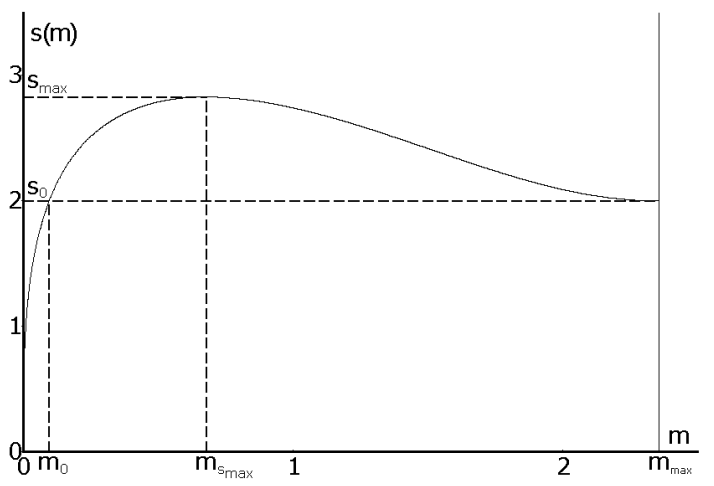

Fig. 6. The dependence of area of the spalling surface on mass of the chipped fragment in case of a concave parabolic "lens".

We assume that if at certain value of $s$ mass of the chipped fragments may have one of $N_{s}$ values, then the probability of spalling of fragment with any of these masses, in condition that the area of split is $s$, the same and equal to $1 / N_{s}$.

Now we find the distribution function $P_{m}(m)$.

1. Consider the region $m \in\left[0, m_{0}\right)$. The graph shows that in the region $m \in\left[0, m_{0}\right)$ function $s(m)$ reversible, so here, as in the cases above, we can find $P_{m}(m)$ as

$$
P_{m}(m)=P_{s}(s(m)), m \in\left[0, m_{0}\right)
$$

2. Consider the region $m \in\left[m_{0}, m_{s_{\max }}\right)$. We select in this region interval of the masses $\left(m_{1}, m_{2}\right)$. This interval of masses corresponds to the interval of split areas $\left(s_{1}, s_{2}\right)$. The probability that the area of split in the fragmentation of the lens gets into interval $\left(s_{1}, s_{2}\right)$ is $P_{s}\left(s_{2}\right)-P_{s}\left(s_{1}\right)$. Since each value of the area in this interval corresponds to two values of the mass, then according to the assumptions made above, the probability of hitting the fragment mass in the interval $\left(m_{1}, m_{2}\right)$ is $\frac{1}{2}\left(P_{s}\left(s_{2}\right)-P_{s}\left(s_{1}\right)\right)$. Thus, in region $m \in\left[m_{0}, m_{s_{\max }}\right)$ fragments distribution on masses have the form

$$
P_{m}(m)=P_{s}\left(s\left(m_{0}\right)\right)+\frac{1}{2}\left(P_{s}(s(m))-P_{s}\left(s\left(m_{0}\right)\right)\right), m \in\left[m_{0}, m_{s_{\max }}\right)
$$

or

$$
P_{m}(m)=\frac{P_{s}(s(m))+P_{s}\left(s\left(m_{0}\right)\right)}{2}, m \in\left[m_{0}, m_{s_{\max }}\right)
$$

3. Finally, let's consider the region $m \in\left[m_{s_{\max }}, m_{\max }\right]$. Arguing as in item 2, we get

$$
P_{m}(m)=\frac{P_{s}\left(s\left(m_{s_{\max }}\right)\right)+P_{s}\left(s\left(m_{0}\right)\right)}{2}+\frac{P_{s}\left(s\left(m_{s_{\max }}\right)\right)-P_{s}(s(m))}{2}, m \in\left[m_{s_{\max }}, m_{\max }\right]
$$

or, taking into account $P_{s}\left(s\left(m_{s_{\max }}\right)\right)=1$

$$
P_{m}(m)=1+\frac{1}{2}\left(P_{s}\left(s\left(m_{0}\right)\right)-P_{s}(s(m))\right), \quad m \in\left[m_{s_{\max }}, m_{\max }\right]
$$

Let's define values of $s_{0}, m_{0}, s_{\max }, m_{s_{\max }}, m_{\max }$. 
Values $s_{\max }$ and $m_{s_{\max }}$ can be found, determining the position of the maximum of expression $s(h)$ and substituting found $h$ in expression $s(h)$ and $m(h)$. From $s(h)=\frac{2}{3} k \sqrt{R^{2}-h^{2}}\left(R^{2}+2 h^{2}\right)$ we obtain

$$
h_{s_{\max }}=R / \sqrt{2}
$$

From where

$$
\begin{gathered}
s_{\max }=\frac{2 \sqrt{2}}{3} k R^{3} \\
m_{s_{\max }}=\frac{k \sigma R^{4}}{2}\left(\frac{\pi}{4}-\frac{1}{3}\right)
\end{gathered}
$$

Further, $s_{0}$ is the area of split at $h=0$,

$$
s_{0}=\frac{2}{3} k R^{3}
$$

However, this area of split occurs and for some other $h=h_{0}$. Mass $m_{0}$ is a mass of fragments, chipped at the $h=h_{0}$. Equating $s(h)=s_{0}$, we obtain

$$
h_{0}=\sqrt{\frac{\sqrt{3}}{2}} R
$$

Thus,

$$
m_{0}=\frac{1}{2} k \sigma R^{4}\left(\frac{\pi}{2}-\arcsin \sqrt{\frac{\sqrt{3}}{2}}-\frac{2}{3} \sqrt{\frac{\sqrt{3}}{2}} \sqrt{1-\frac{\sqrt{3}}{2}}+\frac{1}{12} \sin \left(4 \arcsin \sqrt{\frac{\sqrt{3}}{2}}\right)\right)
$$

Finally,

$$
m_{\max }=m(0)=\frac{\pi}{4} k \sigma R^{4}
$$

Let, as above $P_{s}(s)=A s^{\alpha}, \quad 0<\alpha<1$. As above, we take $\alpha=\frac{1}{2}$. Probability $P_{s}\left(s\left(m_{0}\right)\right)=P_{s}\left(s_{0}\right)$, entering in expression for $P_{m}(m)$ above, is equal to

$$
P_{s}\left(s_{0}\right)=A \sqrt{\frac{2}{3} k R^{3}}
$$

Thus, distribution of the fragments by mass at the fragmentation of the concave "lens" have the form

$$
P_{m}(m)=\left\{\begin{array}{l}
P_{s}(s(m)), m \in\left[0, m_{0}\right) \\
\frac{1}{2}\left(P_{s}(s(m))+P_{s}\left(s\left(m_{0}\right)\right)\right), m \in\left[m_{0}, m_{s_{\max }}\right) \\
\frac{1}{2}\left(1+P_{s}(s(m))\right), m \in\left[m_{s_{\max }}, m_{\max }\right]
\end{array}\right.
$$

Or, for $P_{s}(s)=A s^{\frac{1}{2}}$

$$
P_{m}(m)=\left\{\begin{array}{l}
A s(m)^{\frac{1}{2}}, m \in\left[0, m_{0}\right) \\
\frac{1}{2}\left(A s(m)^{\frac{1}{2}}+A\left(\frac{2}{3} k R^{3}\right)^{\frac{1}{2}}\right), m \in\left[m_{0}, m_{s_{\max }}\right) \\
\frac{1}{2}\left(1+A s(m)^{\frac{1}{2}}\right), m \in\left[m_{s_{\max }}, m_{\max }\right]
\end{array}\right.
$$

at found above $m_{0}, m_{s_{\max }}, m_{\max }$ and pointed above parametrically given function $s(m)$. $A-$ norming factor, equal to

$$
A=\left(s_{\max }\right)^{-\frac{1}{2}}=\left(\frac{2 \sqrt{2}}{3} k R^{3}\right)^{-\frac{1}{2}}
$$



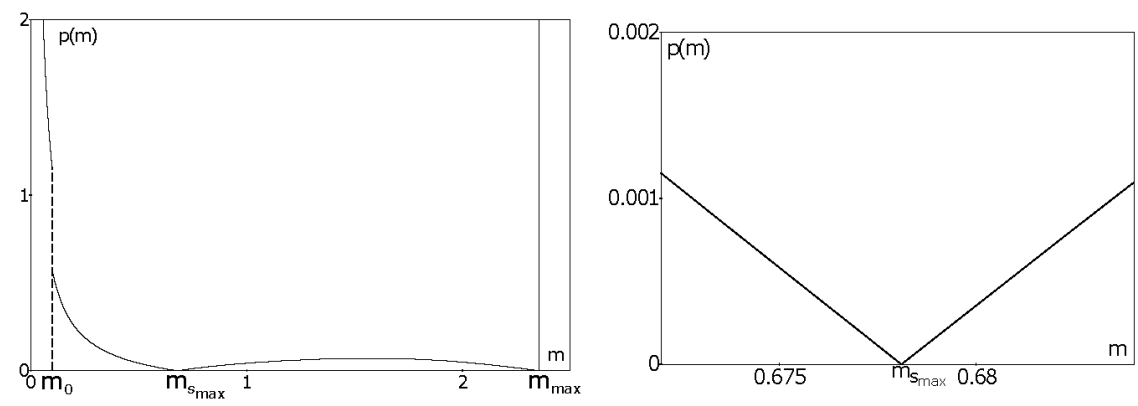

Fig. 7. The distribution density of the fragments by mass at the fragmentation of the concave "lens". Right the behavior of the density near the point $m_{s_{\max }}$ shown separately.

Thus, the probability distribution of formation of fragment mass $m$ is obtained for fragmentation of the concave lens. The probability density $p_{m}(m)$ can be obtained by differentiation of this distribution. Figure 7 shows a graph of the density distribution. The graph obtained by numerical differentiation.

It can see that in points $m=m_{0}$ and $m=m_{s_{\max }}$ the density have exception. When $m=m_{0}$ finite discontinuity take place, when $m=m_{s_{\max }}$ - a break. Indeed, for $m=m_{0}$ mode "one area - one mass" regime replaced by regime "one area - two masses". When $m=m_{s_{\max }}$ similar fragmentation regime change does not occur, and only values of the split area begin to repeat, values, that has taken place at lower masses.

Let's now find the asymptotic behavior of the distribution density. Asymptotic behavior of the function $s(m)$ will be sought by determining the asymptotic behavior of the functions $s(h)$ and $m(h)$. This can easily be done, taking into account that the concave "lens" is a flat "lens" from which convex is "subtracted".

At small masses $m \rightarrow 0, h=R(1-\delta) \rightarrow R$

$$
\begin{gathered}
s(h) \approx 2 \eta R \sqrt{2} \sqrt{\delta}-\frac{4}{3} k R^{3}(2 \delta)^{3 / 2} \approx 2 \eta R \sqrt{2} \sqrt{\delta} \\
m(h) \approx \frac{\sigma R^{2} \eta}{\sqrt{2}} \cdot 2 \frac{2}{3} \cdot \delta^{3 / 2}-\frac{k \sigma R^{4}}{8} \sqrt{2} \times \frac{1039}{120} \delta^{5 / 2} \approx \frac{\sigma R^{2} \eta}{\sqrt{2}} \cdot 2 \frac{2}{3} \cdot \delta^{3 / 2},
\end{gathered}
$$

i.e. coincide with the expressions in the case of "flat lens". So and distribution at small $m$ is equal to

$$
P_{m}(m)=P_{s}(s)=\left(\frac{s}{s_{\max }}\right)^{1 / 2}
$$

And that means, that the asymptotic behavior will be similar to the asymptotic behavior of a flat lens, but with a different value of $s_{\max }$. In the case of a flat lens $s_{\max }=2 \eta R$, in the case of concave $s_{\max }=\frac{2 \sqrt{2}}{3} k R^{3}$ and $\eta=k R^{2}$. Thus,

$$
\begin{gathered}
p_{m}(m)=\frac{\sqrt{2 k R^{3}}}{\sqrt{\frac{2 \sqrt{2}}{3} k R^{3}}} \cdot 2^{1 / 4}\left(\frac{\sigma R^{2} \eta}{\sqrt{2}} \cdot 2 \frac{2}{3}\right)^{-1 / 6} \frac{1}{6} \cdot m^{-5 / 6} \\
p_{m}(m)=\sqrt{3}\left(\frac{\sigma R^{2} \eta}{\sqrt{2}} \cdot 2 \frac{2}{3}\right)^{-1 / 6} \frac{1}{6} \cdot m^{-5 / 6}
\end{gathered}
$$

$\eta$ - thickness at the bound, $\eta=k R^{2}$.

At large masses $m \rightarrow m_{\max }=\frac{\pi}{4} \sigma k R^{4}, h \rightarrow 0$. Let $\frac{h}{R}=\delta$. Then, considering, as above, a concave "lens" as "remainder" of flat and convex, we obtain the asymptotic behavior

$$
m(h(\delta))=\sigma k R^{4}\left(\frac{\pi}{4}-\frac{2}{3} \delta\right)
$$




$$
s(h(\delta))=k R^{3}\left(\frac{2}{3}+\delta^{2}\right)
$$

From where

$$
s(m)=k R^{3}\left(\frac{2}{3}+\frac{9}{4}\left(\frac{\pi}{4}-\frac{m}{\sigma k R^{4}}\right)^{2}\right)
$$

The distribution in this area has the form

$$
P_{m}(m)=1+\frac{1}{2}\left(P_{s}\left(s\left(m_{0}\right)\right)-P_{s}(s(m))\right)
$$

Then the distribution density

$$
p_{m}(m)=-\left.\frac{1}{2} \frac{d P_{s}(s)}{d s}\right|_{s=s(m)} \frac{d s}{d m}
$$

Derivative of split area with respect to fragment mass is

$$
\frac{d s}{d m}=-\frac{9}{2 \sigma R}\left(\frac{\pi}{4}-\frac{m}{\sigma k R^{4}}\right)
$$

For $P_{S}(s)=A s^{\frac{1}{2}}, A=s_{\max }^{-\frac{1}{2}}$

$$
\left.\frac{d P_{s}(s)}{d s}\right|_{s=s(m)}=\frac{1}{2} \frac{1}{\sqrt{s_{\max }}} \frac{1}{\sqrt{s(m)}}
$$

Thus, asymptotics $p_{m}(m)$ have the form

$$
p_{m}(m)=\frac{1}{4} \frac{1}{\sqrt{s_{\max }}} \frac{1}{\sqrt{k R^{3}\left(\frac{2}{3}+\frac{9}{4}\left(\frac{\pi}{4}-\frac{m}{\sigma k R^{4}}\right)^{2}\right)}} \cdot \frac{9}{2 \sigma R}\left(\frac{\pi}{4}-\frac{m}{\sigma k R^{4}}\right)
$$

Or, taking into account $\frac{\pi}{4}-\frac{m}{\sigma k R^{4}}<<1$ and $s_{\max }=\frac{2 \sqrt{2}}{3} k R^{3}$

$$
p_{m}(m)=\frac{27}{16 \cdot 2^{1 / 4}} \frac{1}{k \sigma R^{4}}\left(\frac{\pi}{4}-\frac{m}{\sigma k R^{4}}\right)
$$

The resulting asymptotic behavior for large masses is linear, as in the previous cases.

Graph of density and found above asymptotics is shown in Fig. 8.

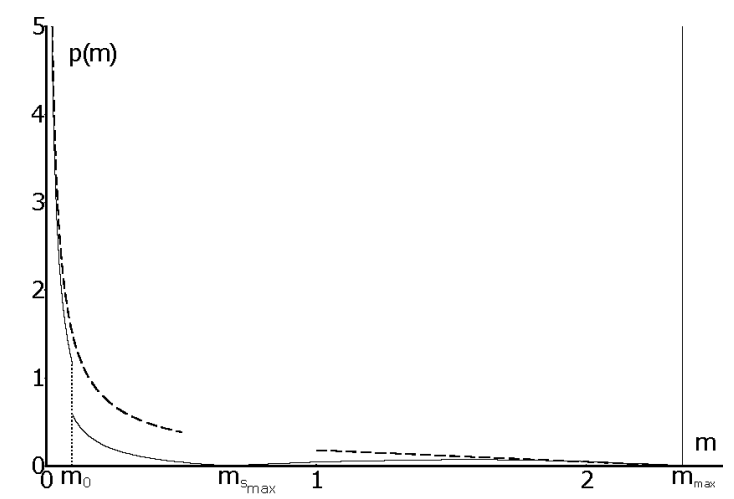

Fig. 8. The distribution density of the fragments by mass at fragmentation of concave parabolic "lens" and the asymptotic behavior of the density. 


\section{Conclusions.}

Fragmentation of three kinds of quasi-two-dimensional round "lenses" are studied - parabolic convex "lens" with the thickness profile $\eta(r)=k\left(R^{2}-r^{2}\right)$, a flat plate of constant thickness $\eta$ and a concave "lens" with the profile $\eta(r)=k r^{2}$. The study was conducted taking into account the dependence of the probability of split on required for this energy, that is, the area of split surface. This dependence has been selected as $P_{s}(s)=A s^{\alpha}, 0<\alpha<1$, where $P_{s}(s)$ - distribution function of split area. Results were as follows.

1. For each case, the distribution function of fragments by mass analytically obtained. The function obtained in parametric form, in the text of work there are graphs (Figure 3, Figure 4, Figure 8) of the density of this distribution.

2. Also, in each case we obtained in an explicit form the asymptotics of the distribution density. These asymptotics are also shown in the graphs (Figure 3, Figure 4, Figure 8) in the text of work. Asymptotics are follows.

For a convex "lens". Large-scale asymptotic behavior

$$
p_{m}(m)=\frac{27}{32} \frac{1}{k \sigma R^{4}}\left(\frac{\pi}{4}-\frac{m}{k \sigma R^{4}}\right)
$$

Small-scale

$$
p_{m}(m)=2^{3 / 4}\left(\frac{k \sigma R^{4}}{8} \frac{1039}{120} \sqrt{2}\right)^{-3 / 10} \cdot \frac{3}{10} m^{-7 / 10}
$$

For a flat "lens". Large-scale asymptotic behavior

$$
p_{m}(m) \approx \frac{1}{8 \sigma \eta R^{2}}\left(\frac{\pi}{2}-\frac{m}{\sigma \eta R^{2}}\right)
$$

Small-scale

$$
p_{m}(m)=2^{1 / 4}\left(\frac{\sigma R^{2} \eta}{\sqrt{2}} \cdot 2 \frac{2}{3}\right)^{-1 / 6} \frac{1}{6} \cdot m^{-5 / 6}
$$

For a concave "lens". Large-scale asymptotic behavior

$$
p_{m}(m)=\frac{27}{16 \cdot 2^{1 / 4}} \frac{1}{k \sigma R^{4}}\left(\frac{\pi}{4}-\frac{m}{\sigma k R^{4}}\right)
$$

Small-scale

$$
p_{m}(m)=2^{1 / 4}\left(\frac{\sigma R^{2} \eta}{\sqrt{2}} \cdot 2 \frac{2}{3}\right)^{-1 / 6} \frac{1}{6} \cdot m^{-5 / 6},
$$

i.e. coincides with the asymptics in the case of a flat plate.

3. It was found that in all cases the large-scale asymptotic behavior is linear and small-scale - a power with a different exponent for different cases.

4. In the study of the fragmentation of the flat plate was also investigated the case corresponding to the one studied in [13], devoted to the fragmentation of plates of zero thickness. In this case, the probability of spalling by a particular chord was introduced not depending on the split area, and on other considerations. Namely, the case of the uniform distribution of crack-chords by distance from the center of the plate was investigated. In this case, the probability distribution of split areas have the form

$$
P_{s}(s)=1-\sqrt{1-\left(\frac{s}{2 \eta R}\right)^{2}}
$$

For this case also obtained the asymptotic behavior of the distribution density of the fragments by mass. They are as follows. 
Large-scale asymptotic behavior

$$
p_{m}(m)=\frac{1}{2 \sigma \eta R^{2}}
$$

Small-scale asymptotics

$$
p_{m}(m)=\left(\frac{1}{2 \sqrt{3} \sigma R^{2} \eta}\right)^{\frac{2}{3}} m^{-\frac{1}{3}}
$$

Obtained expressions coincide with the expressions in [13].

\section{References}

1. V.V.Yanovsky, A.V.Tur, O.V.Kuklina, Zh. Eksp. i Teor. Fiz., 137(4), 1 (2010).

2. P.L.Krapivsky, E.Ben-Naim, Phys.Rev. E., 50, 3502 (1994).

3. D.L.Turcotte, J.Geophys. Res, 91, 1921 (1986).

4. B.R.Lawn, T.R.Wilshaw, Fracture of Brittle Solids. Cambridge University Press, Cambridge (1975).

5. R.Shinnar, J. Fluid Mech., 10, 259 (1961).

6. K.C.Chase, P.Bhattacharyya, A.Z.Mekjian, Phys. Rev. C, 57, 822 (1998).

7. S.Redner, Statistical Models for the Fracture of Disordered Media, ed. H. J. Herrmann and S. Roux. Elsevier Science, New York (1990).

8. T.Ishii, M.Matsushita, J. Phys. Soc. Jap., 61, 3474 (1992).

9. L.Oddershede, P.Dimon, and J.Bohr, Phys. Rev. Lett., 71, 3107 (1993).

10. T.Kadono, Phys. Rev. Lett., 78, 1444 (1997).

11. O.V.Kuklina, V.V.Mozgin, A.V.Tur, V.V.Yanovsky, Functional Materials, 8, 233 (2001).

12. Yu.P.Virchenko, R.Ye.Brodskii, Modern Probl. Nat. Sci., 1, 81 (2014).

13. R.Ye.Brodskii, A.V.Tur, V.V.Yanovsky, Functional materials, 19, 525 (2012). 